\title{
Living donor kidney transplantation: Let's talk about it
}

\author{
Authors: Videha Sharma, ${ }^{\mathrm{A}}$ Reuben Roy, ${ }^{\mathrm{B}}$ Oana Piscoran, ${ }^{\mathrm{A}}$ Angela Summers, ${ }^{\mathrm{C}}$ David van Dellen ${ }^{\mathrm{D}}$ and \\ Titus Augustine ${ }^{\mathrm{E}}$
}

\begin{abstract}
Transplantation is the preferred treatment option for end-stage renal disease as it offers superior results and patient reported outcomes in comparison to dialysis. Patients treated with a transplant live longer, healthier and more independent lives. Transplantation is also more cost-effective, reducing the overall burden of renal disease. Despite the rising incidence of renal failure, the uptake of living donor kidney transplantation has been static across the UK for several years. Among transplantation, living donation offers a number of advantages compared with deceased donor transplantation. The procedure is more likely to be performed pre-dialysis and the elective nature allows for better perioperative planning. Awareness for living donation processes among healthcare professionals, patients and the public appears to be poor. Sharing information regarding the process will help educate colleagues, dispel myths and, crucially, allow patients the opportunity to talk about this treatment option with their hospital doctor.
\end{abstract}

KEYWORDS: Chronic kidney disease, end-stage renal disease, kidney transplantation, organ donation, living donation

DOI: $10.7861 /$ clinmed.2020-0047

\section{Introduction}

Chronic kidney disease (CKD) is an increasing global health issue with over 5 million patients requiring renal replacement therapy (RRT) in the form of dialysis or transplantation worldwide. ${ }^{1}$ In the UK alone, 8,000 new patients started RRT in 2017, a $14 \%$ increase

Authors: ${ }^{\text {A }}$ clinical research fellow in transplantation, Manchester University Hospitals NHS Foundation Trust, Manchester, UK and University of Manchester, Manchester, UK; ${ }^{\mathrm{B}}$ specialty trainee in renal medicine, Manchester University Hospitals NHS Foundation Trust, Manchester, UK; ' ${ }^{C}$ senior research fellow in transplantation, Manchester University Hospitals NHS Foundation Trust, Manchester, UK and University of Manchester, Manchester, UK; ${ }^{D}$ consultant transplant surgeon, Manchester University Hospitals NHS Foundation Trust, Manchester, UK and University of Manchester, Manchester, UK; Eprofessor of clinical transplantation and consultant transplant surgeon, Manchester University Hospitals NHS Foundation Trust, Manchester, UK and University of Manchester, Manchester, UK over the previous 5 years. Only a minority of these patients (9\%) received a transplant as their primary treatment. ${ }^{2}$ The rising prevalence of CKD mandates increased focus on strategies to improve uptake of transplantation to reduce both the overall burden of disease and costs associated with dialysis.

Transplantation is the only treatment that provides a sustained cure for end-stage renal disease (ESRD) and should be the primary treatment of choice for all eligible patients. ${ }^{3}$ Dialysis is the alternative form of RRT. However, it is associated with significant morbidity and increased mortality. ${ }^{4}$ It further impacts on patients quality of life and carries a greater psychological and financial burden. ${ }^{5}$ There are currently over 4,500 patients on the kidney transplant waiting list in the UK and the median waiting time for a kidney is just under 3 years. ${ }^{6}$ Most patients are therefore established on dialysis for some time prior to transplantation. Patients receiving a transplant prior to starting dialysis have improved outcome. Time on dialysis is, therefore, a potentially modifiable risk factor to improve renal transplant and ultimately patient outcomes. ${ }^{\text {? }}$

Living donor kidney transplantation (LDKT) is considered the optimum treatment option for ESRD as it offers the best outcomes in terms of patient and graft survival. The uptake of living donation in the UK has been static over the past 8 years, and has in fact dropped in the $2018 / 2019$ financial year. ${ }^{8}$ LDKT is unique in that, while it is considered the best option for patients with renal failure, it elicits a range of views and opinions among healthcare professionals (HCPs). Poor education among clinicians, patients and entire communities forms one of the main barriers to achieving the required rates in addressing the problem of ESRD. ${ }^{9}$

Most patients receive dialysis in the secondary care (hospital) setting and interact with a wide range of HCPs. As CKD is the sequelae of multiple causative factors, patients are reviewed by different hospital specialists beyond nephrologists, including diabetologists, rheumatologists and geriatricians. As most living donors originate from patients' social networks, non-renal HCPs may be well suited to help overcome some barriers in promoting live donation, dispel myths and, critically, identify potential donors. They may also contribute to the pathway by helping facilitate pre-donation investigations at local hospitals, reducing logistical difficulties and financial implications of travelling to tertiary centres.

\section{Current state of practice}

LDKT offers a number of advantages over deceased donor kidney transplantation (DDKT) and is a well-established practice in the UK. There are 23 centres offering an adult living donor service, 
and transplant activity is overseen by NHS Blood and Transplant (NHSBT) and the Human Tissue Authority (HTA). LDKT is an elective (planned) procedure with a shorter hospital stay and quicker recovery than DDKT. The latter is generally unplanned and therefore may place greater strain on existing healthcare infrastructure. Due to its planned approach, LDKT offers a greater likelihood of patients being transplanted pre-dialysis (38\% for LDKTs vs $13 \%$ for DDKTs). ${ }^{6,8}$

Due to the benefits of transplantation over dialysis, it is important every patient with ESRD requiring RRT be offered a transplant unless contraindicated. In reality, there is significant variation in practice across the UK, with waiting times, pre-emptive transplant rates and percentage of dialysis patients treated with an LDKT differing markedly across the country. ${ }^{2}$ There appears to be little awareness of the referral and work-up process among most non-renal practitioners and junior doctors. ${ }^{10}$ The UK guidelines for LDKT were last updated in 2018 and summarise the evaluation process. The pathway is designed to assess the donor's compatibility to donate to the potential recipient in addition to their overall health. A particular focus is placed on kidney function and cardiovascular risk factors, including hypertension and diabetes to minimise long-term health risks. ${ }^{11}$

The donor assessment process can be daunting and intense, particularly as most donors are previously healthy with little preceding contact with HCPs. Unexpected findings such as previously undiagnosed comorbidities and strain on relationship, or psychological stress, may occasionally arise during the workup process. Regular communication, education and emotional support are needed to minimise drop-out and improve donor experience. ${ }^{12}$ Once the donor-recipient pair are deemed medically and clinically suitable, an interview with an independent assessor is organised to impartially ensure there is no coercion, monetary reward or other ethical contraindication to the donation.

Logistics on the day of surgery are planned together with the medical and nursing team to minimise the time between donor nephrectomy and implantation of the organ. The process is routinely completed in a standard operating day with each procedure taking 2-3 hours of operating time. Donors undergo an enhanced recovery pathway with a hospital stay of 3-4 days and return to previous levels of general activity by 3 months.

\section{Risks to donors}

It is paramount that all HCPs dealing with living donors appreciate that the physical health and psychological wellbeing of the donor take precedence during the entire process. Donors must be provided with correct information regarding risks based on scientific evidence and current practice guidelines, which benefit from increased actuarial probability to guide discussion. They must be allowed appropriate time and dialogue to reach a well-informed decision, which is likely to give them the expected fulfilment of donation. ${ }^{11}$

The maxim that donors must be young, active and fit is being challenged with the perceived risk/benefit potentially greater for well-selected older donors. Human leukocyte antigen / blood group ( $A B O$ ) incompatibility is no longer an absolute contraindication to transplantation due to advances in medical and immunomodulatory strategies. ${ }^{13}$ The UK Living Kidney Sharing Scheme has also allowed donor-recipient pairs to exchange kidneys based on shared compatibility, offering transplantation to patients who previously may have been excluded. Age alone is not an absolute contraindication to donation and the oldest reported living donor was over 80 years of age. Moderate obesity (body mass index of $30-35 \mathrm{~kg} / \mathrm{m}^{2}$ ) and well-controlled hypertension or diabetes mellitus, while requiring thorough and enhanced assessment, also do not absolutely preclude donation. As our understanding of the risks and benefits of donation has improved, the boundaries of acceptance for donors has extended.

A donor nephrectomy is a serious life-changing event, which may take many months of preparation, followed by several months of recovery. This may be a deterrent to younger individuals during their working life and may further mandate a focus on identifying potential donors for whom the impact of surgery has less significant wider ramifications. There are inherent short-term risks; more recently these have been minimised with laparoscopic nephrectomy as the standard technique. With this, the current quoted mortality is low at $0.03 \% .^{11}$

There has been a significant focus on assessing the long-term health risks of kidney donation. Follow-up data from 1963 to 2007 in Norway have suggested an increase in all-cause mortality among living donors when compared with a control group of individuals who would have been eligible to donate. ${ }^{14}$ However, the Developing Education Science and Care for Renal Transplantation in the European States group concluded that long-term risks such as the development of ESRD or hypertension are acceptably low. This view is further strengthened by follow-up data of 3,028 living donors published by NHSBT showing a median creatinine of $93 \mu \mathrm{mol} / \mathrm{L}$ at 10 years post-donation..$^{8,15}$

By selecting those individuals with intrinsic low risk of long-term sequelae from undergoing a nephrectomy, we can continue to justify the principle of living donation. This is especially relevant in the context of the tangible significant benefit of transplantation for recipients, their families and the wider community.

\section{Let's talk about it}

Secondary care remains a major point of healthcare interaction for both patients and their families, and trust in the profession remains high. The opportunity for those in secondary care to promote awareness and encourage discussion around living donation should not be underestimated.

Patient interaction with the live donor team nearly always occurs via the nephrology department. However, the promotion of LDKT need not be limited to these traditional avenues as these patients frequent other specialty clinics. The message that transplantation is the primary treatment option for ESRD with dialysis being a bridge to transplantation should be widely communicated to patients and families. Sowing the seed of the option of LDKT and creation of referral pathways from specialties other than nephrology may facilitate this process. Any pathways should also be highlighted to trusts without nephrology departments as patients in those may be inherently disadvantaged.

Educational and promotional events, in both hospitals and the community, where renal transplant clinicians and/or previous donors and recipients can discuss living donation may be another suitable way to disseminate information and improve access to transplantation. Awareness of such events can be raised by hospital colleagues working in allied specialties. To achieve the best possible outcomes for patients with ESRD and potential donors, the facts need to be shared clearly and unambiguously. 
This would favour pre-emptive LDKT from well-informed, healthscreened donors.

\section{References}

1 Luyckx VA, Tonelli M, Stanifer JW. The global burden of kidney disease and the sustainable development goals. Bull World Health Organ 2018;96:414-22D.

2 Pyart R, Evans KM, Steenkamp R et al. The 21st UK Renal Registry annual report: A summary of analyses of adult data in 2017. Nephron 2020;144:59-66.

3 Schnuelle P, Lorenz D, Trede M, Van Der Woude FJ. Impact of renal cadaveric transplantation on survival in end-stage renal failure: evidence for reduced mortality risk compared with hemodialysis during long-term follow-up. J Am Soc Nephrol 1998;9:2135-41.

4 Oniscu GC, Brown H, Forsythe JL. Impact of cadaveric renal transplantation on survival in patients listed for transplantation. J Am Soc Nephrol 2005;16:1859-65.

5 Laupacis A, Keown P, Pus $\mathrm{N}$ et al. A study of the quality of life and cost-utility of renal transplantation. Kidney Int 1996;50: 235-42.

6 NHS Blood and Transplant. Annual report on kidney transplantation. NHS, 2019.

7 Meier-Kriesche HU, Kaplan B. Waiting time on dialysis as the strongest modifiable risk factor for renal transplant outcomes: a paired donor kidney analysis. Transplantation 2002;74:1377-81.

8 NHS Blood and Transplant. Annual report on living donor kidney transplantation. NHS, 2019.
9 Barnieh L, Collister D, Manns B et al. A scoping review for strategies to increase living kidney donation. Clin J Am Soc Nephrol 2017:12:1518-27.

10 Pruthi R, Tonkin-Crine S, Calestani M et al. Variation in practice patterns for listing patients for renal transplantation in the United Kingdom: A national survey. Transplantation 2018;102:961-8.

11 Andrews PA, Burnapp L, Manas D et al. Summary of the British Transplantation Society/Renal Association UK guidelines for living donor kidney transplantation. Transplantation 2012;93:666-73.

12 Rodrigue JR, Pavlakis M, Danovitch GM et al. Evaluating living kidney donors: relationship types, psychosocial criteria, and consent processes at US transplant programs. Am J Transplant 2007:7:2326-32.

13 Baid-Agrawal S, Frei UA. Living donor renal transplantation: recent developments and perspectives. Nat Clin Pract Nephrol 2007;3: 31-41.

14 Mjøen G, Hallan S, Hartmann A et al. Long-term risks for kidney donors. Kidney Int 2014;86:162-7.

15 Maggiore U, Budde K, Heemann U et al. Long-term risks of kidney living donation: review and position paper by the ERA-EDTA DESCARTES working group. Nephrol Dial Transplant 2017;32: 216-23.

Address for correspondence: Mr Videha Sharma, Department of Renal and Pancreatic Transplantation, Manchester University NHS Foundation Trust, Oxford Road, Manchester M13 9WL, UK.

Email: videha.sharma@postgrad.manchester.ac.uk

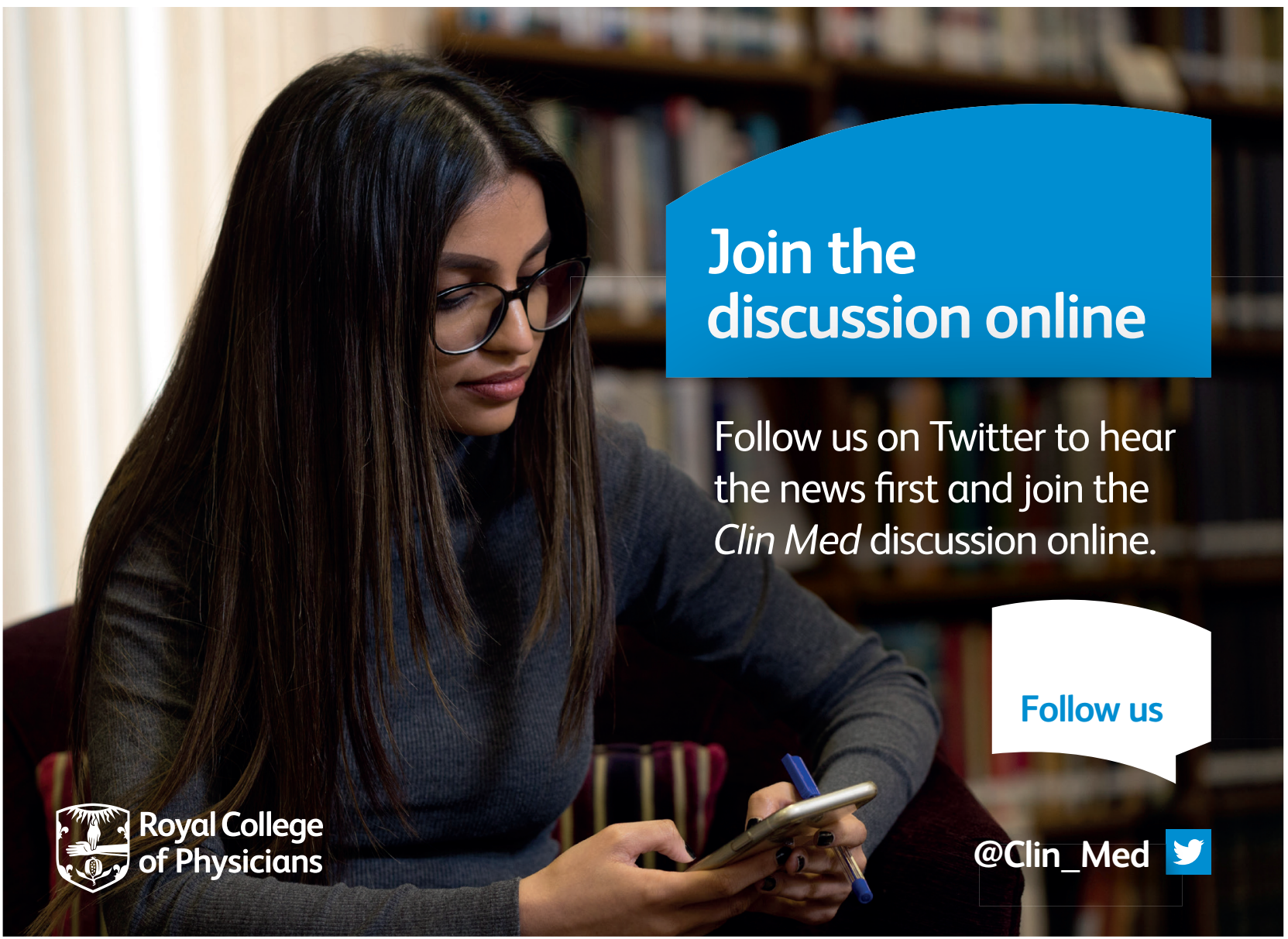

